\title{
Preface to the special issue
}

Fractional Partial Differential Equations (FPDEs) attract more and more attentions in recent years. Fractional calculus is, of course, not new. It was initiated in 1665 in a letter of L'Hospital to Leibnitz while asking for clarification on the meaning of the half derivative of the function $\mathrm{f}(\mathrm{x})=\mathrm{x}$. Nevertheless, impressive progress has been made in the last three decades, and the number of papers devoted to FPDEs increases exponentially. Nowadays, FPDEs have found numerous applications in diverse and widespread fields such as computational finance, fluid mechanics, electrochemistry, biological population models, optics, signal processing, just to name a few.

The aim of this issue is to bring together the leading researchers in the field of applied mathematics to share their recent work on FPDEs. Various topics concerning on fractional differential equations, such as new numerical schemes, fast solvers, and applications in computational finance are discussed in this volume. The papers were selected after careful review by experts in the field and we would like to express our sincere thanks to all authors and referees for their contributions. We also hope that the readers will enjoy the articles presented in this issue.

Special Issue Editors:

Victor Didenko, Southern University of Science and Technology

Tao Tang, Southern University of Science and Technology

Chuanju Xu, Xiamen University

Tao Zhou, AMSS, Chinese Academy of Sciences 